\title{
L'apprentissage en autonomie sur outil multimédia EMATECH
}

\section{Adam Lindsey-Clark}

\section{OpenEdition}

\section{Journals}

Édition électronique

URL : http://journals.openedition.org/asp/3961

DOI : 10.4000/asp.3961

ISSN : 2108-6354

\section{Éditeur}

Groupe d'étude et de recherche en anglais de spécialité

\section{Édition imprimée}

Date de publication : 1 décembre 1995

Pagination : 381-387

ISSN : 1246-8185

\section{Référence électronique}

Adam Lindsey-Clark, "L'apprentissage en autonomie sur outil multimédia EMATECH », ASp [En ligne] 7-10 | 1995, mis en ligne le 15 novembre 2013, consulté le 21 avril 2019. URL : http:// journals.openedition.org/asp/3961 ; DOI : 10.4000/asp.3961

Ce document a été généré automatiquement le 21 avril 2019

Tous droits réservés 


\title{
L'apprentissage en autonomie sur outil multimédia EMATECH
}

\author{
Adam Lindsey-Clark
}

1 Nous avons au moins deux mots à définir : EMATECH et autonomie. Commençons par le plus simple : EMATECH. Il s'agit d'un logiciel, conçu et développé par l'équipe multimédia et les enseignants de l'École des Mines d'Alès, qui tourne sur micro-ordinateurs équipés de cartes son. Il peut fonctionner en réseau (interne ou externe à l'établissement) ou en postes individuels. EMATECH a remplacé nos vieux laboratoires de langues et est en service depuis deux ans. Nous disposons de deux salles, de quatorze postes chacune, utilisées par les élèves de l'école et par les stagiaires de Formation continue, soit avec un tuteur présent, soit en autonomie guidée, ou en libre service.

2 Le premier module est un système d'apprentissage multimédia, c'est à dire intégrant des sons, des textes et des images, y compris de la vidéo. L'apprenant peut sélectionner les séquences qu'il désire travailler à partir d'une série de lignes sur l'écran de son ordinateur.

3 Le deuxième module est un gestionnaire de travail pour le tuteur. Entre ces deux modules, il y a une messagerie. Il y a aussi un module auteur pour la rapide création de supports pédagogiques multimédia, ou bien la «multimédiatisation» de supports existants.

4 L'ensemble est exceptionnellement convivial et ne demande aucune connaissance de l'informatique, même pour la création de cours multimédia.

5 Première question : qu'est-ce que cet outil nous apporte?

6 Le tableau 1 résume des avantages d'Ematech par rapport à un outil classique.

Tableau 1

\begin{tabular}{|l|l|l|l|}
\hline & Module & Avantages & Fonctionnalités \\
\hline
\end{tabular}




\begin{tabular}{|c|c|c|c|}
\hline $\begin{array}{l}\text { Environnement } \\
\text { d'apprentissage }\end{array}$ & EMATHEQUE & $\begin{array}{l}\text { Laboratoire de langues/ } \\
\text { magnétophone/scope } \\
\text { + multimédia } \\
\text { + visualisation du déroulement } \\
\text { + vue globale } \\
\text { + accès très simple à la bonne } \\
\text { phrase }\end{array}$ & $\begin{array}{l}\text { - texte masquable } \\
\text { d'images } \\
\text { - vidéo } \\
\text { - QCM (texte) } \\
\text { - QCM (vocale) } \\
\text { - QCM (images) } \\
\text { - glossaire }\end{array}$ \\
\hline $\begin{array}{l}\text { Moyen de } \\
\text { communication }\end{array}$ & Messagerie & $\begin{array}{l}\text { + message contextuel } \\
\text { + pas d'attente } \\
\text { + pas d'interruption }\end{array}$ & RNIS/Numeris \\
\hline $\begin{array}{l}\text { Gestionnaire } \\
\text { de travail }\end{array}$ & $\begin{array}{l}\text { EMATECH } \\
\text { tuteur }\end{array}$ & $\begin{array}{l}\text { + vue globale } \\
\text { + accès très simple à la bonne } \\
\text { phrase } \\
\text { + souplesse de programme de } \\
\text { travail } \\
\text { + tutorat à distance ou différé }\end{array}$ & RNIS/Numeris \\
\hline $\begin{array}{l}\text { «Traitement } \\
\text { de cours» }\end{array}$ & $\begin{array}{l}\text { EMATECH } \\
\text { auteur }\end{array}$ & $\begin{array}{l}\text { Transposition } \\
\text { Adaptation } \\
\text { Création }\end{array}$ & $\begin{array}{l}\text { Cours EMA (Ang) } \\
\text { Revue BABEL (4L) } \\
\text { Méthodes (bientôt) }\end{array}$ \\
\hline
\end{tabular}

7 L'outil présente de nombreux avantages; il peut surtout favoriser l'autonomie de l'apprenant.

\section{Autonomie}

N'étant pas complètement autonome, je consulte d'abord les dictionnaires.

Autonomous: independent, not a mere form or state of some other organism ( Shorter Oxford)

Autonomie : droit de se gouverner par ses propres lois (Petit Robert)

droit pour l'individu de déterminer librement les règles auxquelles il se soumet (Kant «L'autonomie de la volonté ») (Petit Robert)

distance que peut franchir un véhicule, un avion, un navire [un apprenant] sans être ravitaillé en carburant (Petit Robert)

9 Des définitions, on pourrait dire, peu adaptées à notre sens du terme. Mais ces quatre définitions présentent quatre notions très pertinentes.

Dans un apprentissage en autonomie, le travail de l'apprenant est centré sur son propre processus d'apprentissage. (Hutchinson \& Waters 1987) ; c'est l'apprenant qui décide ce qu'il fait. (Holec 1980) ; c'est l'apprenant, en examinant ses propres besoins, qui décide quel régime à suivre (Gremmo and Abé 1985) ? Il faut quand même ajouter du carburant de temps en temps (de préférence sur la demande de l'apprenant). 
11 Il y a trois ans quand nous avons commencé ce travail nous nous sommes posé les questions suivantes : est-ce que l'autonomie est souhaitable? Jusqu'à quelle limite ? Estce qu'une grande quantité d'autonomie est réalisable? Quand ? Comment? Pour et par quelles démarches?

12 Au niveau de la quantité d'autonomie souhaitable, notre réponse est simple. Nos élèves et nos stagiaires de Formation continue étaient trop dépendants de leurs enseignants. Par conséquent les élèves ne font pas suffisamment de travail personnel et alors sont relativement faibles en aspects de la langue anglaise qu'ils pourraient théoriquement étudier seuls. Même si, au bout d'un certain temps à l'école, ils arrivent très bien, pour la plupart, à comprendre et à se faire comprendre, ils ont toujours des lacunes, surtout en grammaire et en expression écrite.

Quant à la Formation continue, le fait que les stagiaires ont tendance à ne travailler qu'avec leur enseignant augmente le coût de leur formation. Les entreprises de la région voulaient un type de formation ou les stagiaires feraient plus de travail individuel.

14 Nous avons donc un objectif simple, clair et pratique: réduire la dépendance de l'apprenant par rapport au formateur en ce qui concerne le travail qu'il est complètement capable (théoriquement) de faire seul.

15 Jusqu'à quelle limite peut-on développer cette démarche ? C'est impossible à dire, mais de toute façon on est toujours très loin de cette limite et il n'y a pas de risque de la dépasser pour l'instant.

16 La démarche est-elle réalisable? Nous sommes en train de le découvrir, mais nous savons qu'il faut mettre en marche autant de moyens que possible.

17 Quand cela se fera-t-il ? Pas demain. Nous allons vers l'autonomie. Il y a deux risques si on va trop vite. Si on change tout subitement, on ne saura pas ensuite quelles démarches ont favorisé les changements qu'on constate. Dans un monde idéal, on suivrait une méthodologie scientifique, procédant étape par étape en ne modifiant qu'un paramètre à la fois et en testant rigoureusement l'efficacité de chaque modification. Il est essentiel d'intégrer les diverses démarches dans une politique cohérente. À Alès, le multimédia n'est qu'un des dispositifs qui favorisent l'autonomie.

18 Nous allons en présenter trois : ceux qui concernent et les langues et les nouvelles technologies, donc EMATECH.

\section{Un programme de formation continue en autonomie tutorée à distance}

19 Avec un branchement RNIS/Numeris entre le poste de l'apprenant à son lieu de travail et celui de son tuteur à l'école, EMATECH permet un dialogue entre les deux. En plus, l'enseignant peut regarder le travail du stagiaire, lui envoyer de l'aide, des corrections, des conseils, et bien sûr de nouveaux supports pédagogiques. À l'EMA on met en place un système de formation qui exploite ces possibilités.

20 Le travail des stagiaires (niveau perfectionnement) est découpé en blocs thématiques qui durent environ un mois chacun. Voici une liste des blocs prévus, fondés sur nos programmes de l'école pour pouvoir facilement $\mathrm{y}$ intégrer toutes nos ressources pédagogiques. 
Blocs prévus

\begin{tabular}{|l|}
\hline INTRODUCTION \\
\hline PERSONAL IDENTITY \\
\hline WORK 1 (GENERAL) \\
\hline TRAVEL \\
\hline WORK 2 (SPECIFIC) \\
\hline
\end{tabular}

21 Chaque bloc commence avec un contrôle pour évaluer l'apprenant dans les compétences communicatives qui concernent le thème et identifier les domaines où il a des lacunes. Le contrôle est suivi d'un positionnement où l'enseignant et l'apprenant décident ensemble les objectifs du travail personnel pendant le bloc, ainsi que les ressources disponibles pour les réaliser. Ils créent donc créent un programme de travail personnel comprenant des leçons EMATECH, exercices de grammaire, etc. faisant appel à tous les supports pédagogiques disponibles à l'École.

Pendant la durée du bloc ce programme de travail reste modifiable en fonction des besoins de l'apprenant. Ces modifications peuvent se faire en utilisant la messagerie d'EMATECH.

Les stagiaires se réunissent régulièrement en groupes d'entre trois et cinq pour des cours de conversation sur le thème du bloc et un suivi présentiel de leur travail.

La durée de chaque bloc se termine par un deuxième contrôle pour évaluer les progrès de l'apprenant dans les domaines traités, vérifier l'efficacité du système et donner au stagiaire du retour sur son travail.

En novembre 1994 on a commencé ce programme de formation avec un groupe pilote de six stagiaires de la société Merlin Gérin, qui a une usine à Alès. On constate que les stagiaires sont très divers au niveau de leur autonomie et leurs préférences. Il y en a qui travaillent beaucoup en autonomie. Il y en a qui ne travaillent qu'en cours.

On a rencontré plusieurs problèmes. Ils sont dus à :

- la disponibilité des stagiaires : il est difficile de fixer des rendez-vous réguliers nécessaires pour maintenir la motivation

- l'organisation de l'ensemble : à cause des problèmes de la disponibilité et de la nouveauté des structures de gestion. Résultat : les blocs deviennent trop longs et l'apprenant perd la vue globale de son travail.

- la mise en place du matériel, notamment prises Numeris, ordinateurs suffisamment puissants.

Pourtant, on a fait le bilan de la période pilote en janvier 1995 et Merlin Gérin a augmenté le nombre de ses stagiaires. Nous sommes persuadés que le fait d'avoir plus de personnes impliquées va faciliter la création de groupes homogènes et réduire les problèmes de disponibilité et de mise en place des structures et du matériel. D'autres entreprises de la région d'Alès y participent aussi. 


\subsection{Laboratoire de langues} peu près une heure tous les quatre cours. L'enseignant leur donne un programme de travail de drills ou d'exercices de compréhension auditive. La quantité d'autonomie dans un tel travail est évidemment très limitée, mais encore une fois on constate de la diversité. La plupart des enseignants sont assez directifs; ils imposent le travail, ils écoutent les élèves pour identifier leurs erreurs et les aider. D'autres proposent un programme de travail plus souple, demandent aux élèves de poser les questions s'ils se trouvent en difficulté et s'occupent moins de la correction et du suivi. L'avantage de cette approche est le fait que l'enseignant est plus disponible pour ceux qui ont vraiment besoin de lui. Il peut même créer un travail supplémentaire pour ces élèves.

\subsection{Libre-service}

Nos cours sur EMATECH, ainsi que d'autres logiciels sur la grammaire et les examens First Certificate et TOEFL, sont disponibles en libre-service en dehors des heures de cours. On constate que les élèves et le personnel de l'école qui en profitent sont beaucoup plus nombreux quand ils ont un objectif fixe et proche, par exemple s'ils vont passer First Certificate ou s'ils ressentent un besoin très important comme celui des stagiaires brésiliens qui viennent à Alès en été et qui ont beaucoup de difficulté à suivre leurs cours en français sur les techniques minières.

Afin de tester comment le libre-service peut apporter encore plus à l'apprenant je propose un essai : avoir la présence d'un enseignant pendant une partie du libre-service pour répondre aux questions de chaque apprenant et le guider. On sait combien ce guidage, tel qu'il est pratiqué, par exemple, au SAPAG de l'Université Bordeaux 2, se révèle efficace.

Évidemment, il faut continuer à essayer de toujours fournir de nouveaux supports pédagogiques aussi stimulants, attrayants, ludiques et variés que possible.

La partie théorique de cette communication ainsi que les constatations de la partie descriptive nous mènent aux mêmes conclusions : on ne peut qu'aller vers l'autonomie. Il y a beaucoup de diversité parmi les enseignants et les apprenants. De multiples moyens doivent être utilisés; les nouvelles technologies doivent faire partie d'un dispositif cohérent. Toutes les démarches doivent être intégrées dans ce dispositif. Il faut prévoir du carburant (contact humain, feed-back, etc.). Don't throw the baby out with the bath water: notre plus grande ressource est toujours notre équipe de formateurs.

\section{BIBLIOGRAPHIE}

Hutchinson, T and A. Waters. 1987. English for Specific Purposes: A learning-centred approach. Londres : Cambridge University Press.

ASp, 7-10| 2012 
Holec, H. 1980. Autonomy and Foreign Language Learning. Oxford : Pergamon.

Gremmo M.J. et D. Abe. 1985. « Teaching learning: Redefining the teacher's role ». In Riley, P. (dir.), Discourse and Learning. Londres/New York Longman.

\section{RÉSUMÉS}

Depuis trois ans, l'École des Mines d'Alès développe EMATECH, un logiciel d'apprentissage de langues et d'autres matières. Ce produit étant maintenant très performant, la prochaine étape est d'assurer sa bonne intégration dans plusieurs démarches de formation, y compris des programmes d'apprentissage en autonomie tutoré à distance. L'auteur décrit des expériences de l'EMA dans ces nouveaux domaines. Nos préoccupations concernent les proportions d'ingrédients classiques ou innovants à inclure dans la nouvelle recette afin de pouvoir proposer une formation performante et motivante à long terme. Comment favoriser l'autonomie? Quels sont les points forts de la formation classique? Comment modifier le rôle de l'enseignant sans perdre les apports de son savoir-faire?

Over the last three years, the École des Mines d'Alès has developed EMATECH, a multimedia environment for the learning and teaching of languages and other subjects. The product now works very well. The moment has come to integrate it into various educational projects, including a distance learning programme. The author describes the school's experiences in these new fields. We are concerned with finding the correct proportion of traditional and innovative ingredients to include in the new recipe in order to be able to offer effective learning programmes and long-term motivation. How can learner-autonomy be encouraged? What are the advantages of traditional teaching? How can the teacher's role be modified without losing the fruits of his/her experience and expertise?

\section{INDEX}

Mots-clés : autonomie, formation continue, multimédia, tutorat à distance

Keywords : autonomy, continuing education, distance tutoring

\section{AUTEUR}

\section{ADAM LINDSEY-CLARK}

Adam Lindsey-Clark travaille à l'École des Mines d'Alès depuis septembre 1989. Il est très impliqué dans le multimédia, surtout dans le développement et la mise en œuvre de l'outil EMATECH. Il a fait plusieurs communications sur le multimédia, l'EAD et l'autonomie. Il a douze ans d'expérience de l'enseignement, en matières scientifiques ainsi qu'en anglais. Sur le plan créatif, il est l'auteur de plusieurs supports multimédia. adam.lindsey-clark@ema.fr 